\title{
Risk factors for erysipelas of the leg (cellulitis): case-control study
}

\author{
Alain Dupuy, Hakima Benchikhi, Jean-Claude Roujeau, Philippe Bernard, Loïc Vaillant, \\ Olivier Chosidow, Bruno Sassolas, Jean-Claude Guillaume, Jean-Jacques Grob, Sylvie Bastuji-Garin
}

\begin{abstract}
Objective To assess risk factors for erysipelas of the leg (cellulitis).

Design Case-control study.

Setting 7 hospital centres in France.

Subjects 167 patients admitted to hospital for erysipelas of the leg and 294 controls.

Results In multivariate analysis, a disruption of the cutaneous barrier (leg ulcer, wound, fissurated toe-web intertrigo, pressure ulcer, or leg dermatosis) (odds ratio $23.8,95 \%$ confidence interval 10.7 to 52.5 ), lymphoedema (71.2, 5.6 to 908$)$, venous insufficiency (2.9, 1.0 to 8.7), leg oedema (2.5, 1.2 to 5.1) and being overweight (2.0, 1.1 to 3.7$)$ were independently associated with erysipelas of the leg. No association was observed with diabetes, alcohol, or smoking. Population attributable risk for toe-web intertrigo was $61 \%$.

Conclusion This first case-control study highlights the major role of local risk factors (mainly lymphoedema and site of entry) in erysipelas of the leg. From a public health perspective, detecting and treating toe-web intertrigo should be evaluated in the secondary prevention of erysipelas of the leg.
\end{abstract}

\section{Introduction}

Commonly caused by streptococci, erysipelas is an infectious condition of the skin or subcutaneous tissue, which usually affects the leg (cellulitis). ${ }^{1-3}$ Although a potentially serious disease, erysipelas of the leg can be controlled with antibiotics. As recurrences of erysipelas are common and patients are usually admitted to hospital, cost is an important issue. The identification of risk factors for erysipelas is therefore critical in prevention of the disease.

Several factors, either local (for example, disruption of the cutaneous barrier, lymphoedema, venous insufficiency) or general (for example, diabetes mellitus, overweight, alcohol misuse), have been suspected as risk factors for erysipelas of the leg from a few case series. ${ }^{4-8}$ Owing to the inherent methodological limitations of such studies, however, these factors could not be assessed quantitatively-that is, compared with a control group. We conducted a case-control sudy to assess risk factors for erysipelas of the leg, particularly the role of toe-web intertrigo and other potential sites of entry.

\section{Subjects and methods}

\section{Study design}

We conducted our case-control study prospectively from June 1995 to October 1996 in seven hospital centres in France. Cases and controls were matched for age (range 5 years), sex, and hospital (admission within the same 2 month period). For a type 1 error of $0.05 \%$,
130 cases were sufficient to detect, with a power of $80 \%$, odds ratios $>3.2$ for factors with a prevalence of $5 \%$ in the general population (for example, venous insufficiency) or odds ratios $>2.5$ for factors with a prevalence of $10 \%$ in the general population (for example, toe-web intertrigo).

\section{Cases}

We included patients admitted consecutively to each of the participating centres for erysipelas of the leg. We excluded patients under 15 years of age and patients with abscess or necrotising fasciitis (defined by frank cutaneous necrosis on physical examination or fascial oedema and necrosis detected at surgery). Erysipelas was defined as the sudden onset $(<24$ hours) of a well demarcated cutaneous inflammation, with fever $>38^{\circ} \mathrm{C}$ or chills. Our definition for erysipelas-that is, an acute bacterial dermohypodermatitis-corresponds to non-necrotising cellulitis in other countries or reports. Of the 178 patients recruited, $11(6 \%)$ did not fulfil the inclusion criteria ( 8 had no fever or chills, and 3 had cellulitis elsewhere). The 167 cases comprised 87 men $(52 \%)$ and 80 women (48\%) (mean age 56.5 (SE 1.8) years). The right leg was affected in 85 cases $(51 \%)$, the left in $78(47 \%)$, and both in $4(2 \%)$. Overall, 129 patients $(77 \%)$ were admitted for newly diagnosed erysipelas of the leg, $8(5 \%)$ for a first recurrence, $15(9 \%)$ for a second recurrence, and $15(9 \%)$ for a third or more recurrence.

\section{Controls}

We included two controls for each case matched for age, sex, and hospital, who were admitted for an acute condition not a priori related to one of the suspected risk factors nor related to a chronic disease. Among 323 potential controls, $21(7 \%)$ were excluded because they did not fulfil the above criteria. The 294 controls comprised 154 men (52\%) and 140 women (48\%) (mean age 56.6 (1.1) years) who had been admitted for trauma (109, 37\%), dermatological conditions (49, $17 \%)$, abdominal surgery $(38,13 \%)$, infection $(30,10 \%)$, orthopaedic surgery $(13,4 \%)$, vascular disease $(6,2 \%)$, sciatalgia $(6,2 \%)$, eye disease $(2,1 \%)$, and other conditions $(41,14 \%)$.

\section{Data collection}

One dermatologist in each centre conducted direct interviews with a structured questionnaire and performed the clinical examination of cases and controls. Besides age, sex, and current or past occupation, we assessed general and local potential risk factors. General risk factors included being overweight ( $>120 \%$ of the ideal weight as calculated by Lorentz's formula), diabetes mellitus, smoking (current smoker $v$ non-smoker or past smoker), alcohol misuse (two items on the CAGE questionnaire ${ }^{9}$ ), and seated position at work. Local risk factors were a history of leg surgery, $x$

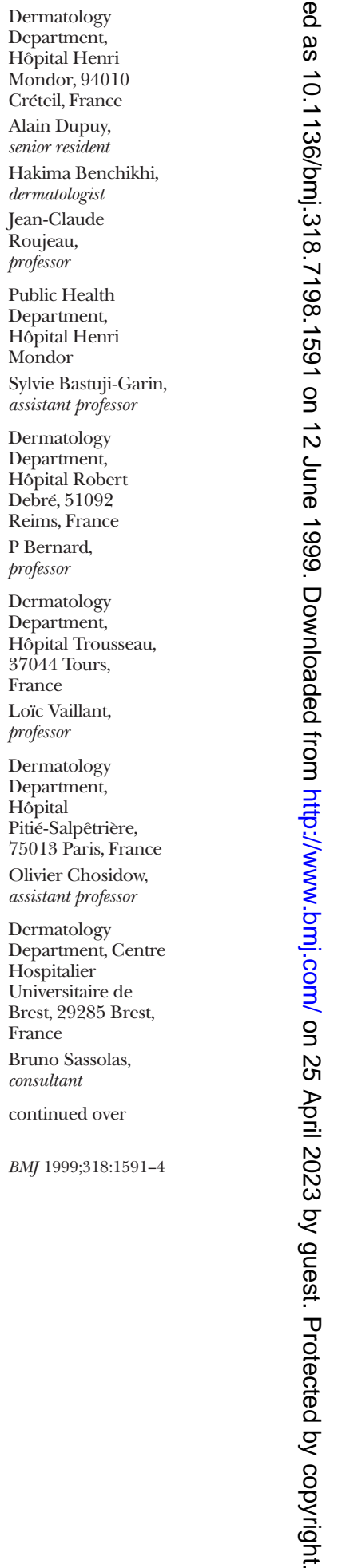


Dermatology Department, Hôpital Pasteur, 68024 Colmar, France

Jean-Claude

Guillaume,

consultant

Dermatology

Department,

Hôpital

Sainte-Marguerite,

13009 Marseille,

France professor

Correspondence to: Dr Bastuji-Garin

sylvie.bastuji-garin@

hmn.ap-hop-paris.fr
Jean-Jacques Grob,

ray therapy (inferior limb or pelvis), neurological disorders, leg thrombophlebitis, and leg ulcer. Leg oedema, lymphoedema, leg ulcer, pressure ulcer, leg dermatosis, toe-web intertrigo, varicose veins or varicosities, and peripheral pulses were detected by clinical examination. No laboratory investigations were performed.

\section{Data analysis}

We compared both general and local factors between cases and controls.

Venous insufficiency was defined as the presence of at least one of the following: history of either venous leg ulcer or leg phlebitis, or specific dermatitis. A site of entry was considered present if either a leg ulcer, a wound, an excoriated leg dermatosis, a pressure ulcer, or a toe-web intertrigo was present on a leg-whether or not erysipelas was present. Toe-web interspaces were evaluated as: 1, normal; 2, doubtful; 3, abnormal; or 4 , fissurated. We considered intertrigo present with scores of 3 or 4 and absent with scores of 1 or 2 .

In the analysis we included only cases with newly diagnosed erysipelas of the leg (129 patients). ${ }^{10}$ We retained the controls matched to recurrent cases for the unconditional analysis but discarded them for the conditional analysis. As the results of both analyses were similar, we present only the results of the unconditional analysis.

We conducted a standard case-control analysis. ${ }^{10}$ For each exposure we calculated odds ratios and 95\% confidence intervals separately. We used unconditional logistic regression models and forced the matching variables into all models. For lateralised factors, we took into account only the ipsilateral side.

The factors we chose for inclusion in the multivariate model were selected by using multiple $2 \times 2$ analyses on those variables that emerged from the univariate analysis, and we assessed interaction and confounding by fitting multiplicative models. We then conducted a final backward step by step regression.

Table 1 Univariate analysis of risk factors for erysipelas of the leg

\begin{tabular}{|c|c|c|c|}
\hline Risk factors & $\begin{array}{c}\text { No }(\%) \text { of } \\
\text { cases }(n=129)\end{array}$ & $\begin{array}{c}\text { No }(\%) \text { of } \\
\text { controls }(n=294)\end{array}$ & $\begin{array}{c}\text { Odds ratio* } \\
(95 \% \mathrm{CI})\end{array}$ \\
\hline \multicolumn{4}{|l|}{ General } \\
\hline Overweight & $68(53)$ & $97(33)$ & 2.5 (1.6 to 3.9$)$ \\
\hline Seated position & $13(11)$ & $26(9)$ & $1.0(0.5$ to 2.0$)$ \\
\hline Diabetes mellitus & $16(13)$ & $24(8)$ & 1.7 (0.8 to 3.5$)$ \\
\hline Alcohol misuse & $11(9)$ & $29(10)$ & $0.9(0.4$ to 2.0$)$ \\
\hline Smoking & $26(20)$ & $77(26)$ & $0.6(0.3$ to 1.2$)$ \\
\hline \multicolumn{4}{|l|}{ Local risk factors } \\
\hline Leg oedema & $48(38)$ & $44(15)$ & $3.6(2.2$ to 6.0$)$ \\
\hline Varicosities & $55(43)$ & $110(38)$ & $1.5(0.9$ to 2.5$)$ \\
\hline \multicolumn{4}{|l|}{ History of: } \\
\hline Phlebitis & $9(13)$ & $6(2)$ & 4.1 (1.4 to 11.6$)$ \\
\hline Leg ulcer & $15(13)$ & $5(2)$ & 8.3 (3.2 to 21.6$)$ \\
\hline Leg surgery & $36(30)$ & $41(15)$ & 2.7 (1.6 to 4.6$)$ \\
\hline Neurological disorder & $13(10)$ & $12(4)$ & $2.1(0.9$ to 5.0$)$ \\
\hline$x$ ray therapy & $5(4)$ & $5(2)$ & $1.7(0.5$ to 5.8$)$ \\
\hline Lymphoedema & $22(18)$ & $1(0.4)$ & 57.7 (16.9 to 197$)$ \\
\hline Abolition of a peripheral pulse & $36(30)$ & $36(13)$ & $2.8(1.5$ to 4.9$)$ \\
\hline Leg ulcer & $17(14)$ & $2(1)$ & 20.6 (6.7 to 63.0$)$ \\
\hline Wound & $47(38)$ & $21(8)$ & $6.8(4.0$ to 11.7$)$ \\
\hline Pressure ulcer & $5(4)$ & $2(1)$ & $6.0(1.4$ to 26.0$)$ \\
\hline Leg excoriated dermatosis & $11(9)$ & $7(3)$ & 3.6 (1.4 to 9.2$)$ \\
\hline Toe-web intertrigo & $83(66)$ & $65(23)$ & 6.6 (4.2 to 10.5$)$ \\
\hline
\end{tabular}

Table 2 Multivariate analysis of risk factors for erysipelas of the leg

Risk factor

Odds ratio* $(95 \% \mathrm{Cl})$

\begin{tabular}{lc}
\hline Lymphoedema & $71.2(5.6$ to 908$)$ \\
\hline Site of entry & $23.8(10.7$ to 52.5$)$ \\
\hline Leg oedema $\dagger$ & $2.5(1.2$ to 5.1$)$ \\
\hline Venous insufficiency & $2.9(1.0$ to 8.7$)$ \\
\hline Overweight & $2(1.1$ to 3.7$)$
\end{tabular}

*Adjusted for age, sex, hospital, and variables in table.

tExcluding oedema related to venous insufficiency.

We conducted specific analyses for lateralised factors. These were defined as local factors that could be present on a patient's limb yet absent on the otherthat is, history of phlebitis, leg ulcer, leg surgery, neurological disorders, $x$ ray therapy, current lymphoedema, abolition of a peripheral pulse, and site of entry (leg ulcer, wound, pressure ulcer, excoriated leg dermatosis, toe-web intertrigo). With the hypothesis that a lateralised factor may be a site of entry if situated on the affected leg, we recorded these factors as ipsilateral (affected side) or contralateral (healthy leg) for cases. For controls, we arbitrarily determined an ipsilateral and a contralateral side in each patient thus allowing comparisons between cases and controls. We also compared ipsilateral and contralateral sides in cases by paired analysis.

In the interests of public health, we calculated population attributable risks as the fraction of the total disease experienced in the population that would not have occurred if the effect associated with the risk factor was absent. This took into account adjusted odds ratios and distribution of exposure among cases. ${ }^{11}$

We analysed the data with SAS-PC (version 6.12, SAS Institute, Cary, NC) and BMDP software (University of California, Berkeley).

\section{Results}

\section{Risk factors for erysipelas of the leg}

In the univariate analysis, seated position at work, diabetes mellitus, alcohol misuse, and smoking were not associated with erysipelas of the leg (table 1), and these were not further analysed. We observed no association with a history of $x$ ray therapy. The associations between erysipelas of the leg and the presence of either varicosities or a history of neurological disorders were close to significance.

Table 2 summarises the results of the multivariate analysis. Lymphoedema was the most prominent risk factor; the presence of a site of entry was also a strong risk factor. The risks associated with leg oedema and venous insufficiency were weaker; overweight was the only general risk factor associated with erysipelas of the leg.

The only two factors of significance between the cases admitted for recurrence and those admitted for a first episode were that patients admitted for recurrence were older (60.3 (2.4) v 56.5 (1.8)) and had a more frequent history of leg surgery $(2.2 ; 1.1$ to 4.7$)$.

\section{Lateralised factors}

The analysis comparing both legs among cases showed that all the factors were more frequently present on the ipsilateral leg than on the contralateral leg, and statisti- 
cal significance was reached only for history of phlebitis and history of $x$ ray therapy (data not shown).

Table 3 compares the number of intertrigo in cases and controls. Before adjustment for number of toe-web intertrigo on the opposite foot, a significant risk was observed on both sides for one intertrigo, and this increased with the number of affected interspaces. After adjustment this relation remained unchanged on the ipsilateral side and disappeared on the contralateral side.

\section{Risks associated with the site of entry}

Site of entry was a strong risk factor for erysipelas of the leg $(24.5 ; 11.0$ to 54.9$)$. We calculated multivariate estimates of odds ratios and population attributable risks associated with each type of site of entry. Leg ulcer (62.5; 7.0 to 556$)$, toe-web intertrigo ( $13.9 ; 7.2$ to 27.0$)$, and traumatic wound $(10.7 ; 4.8$ to 23.8$)$ exhibited strong and highly significant associations with erysipelas of the leg whereas pressure ulcer and excoriated leg dermatosis were not significant. The strongest odds ratio was for leg ulcer, although the population attributable risks associated with leg ulcer (14\%) were much smaller than for intertrigo $(61 \%)$ or wound $(35 \%)$.

\section{Discussion}

To our knowledge this is the first controlled study to examine risk factors for erysipelas of the leg. In our study, diabetes and alcohol misuse were not associated with erysipelas of the leg, and being overweight was the only general factor associated with the condition. We showed that lymphoedema and a site of entry were the main risk factors. Among the different potential sites of entry, toe-web intertrigo had the highest population attributable risk.

Our study has some limitations. Firstly, because our study was hospital based the recruitment of cases could be biased toward more severe disease or more disabled patients. But because no community based study of erysipelas of the leg is available, it was impossible for us to assess whether our cases had more specific risk factors than those patients not referred to hospital. We only assessed patients from dermatology units, and in some hospitals not all cases of erysipelas of the leg are admitted to such units, so referral bias due to concurrent dermatological conditions may have occurred. We do not, however, believe that toe-web intertrigo was a reason for referring patients with erysipelas of the leg to a dermatology unit. Hospital controls were chosen for logistic reasons as we believe that non-inclusion of patients admitted to hospital for a chronic disease or for a disease that could have been a priori related to a suspected risk factor, and recruitment from different surgical or medical units, were sufficient for obtaining an appropriate control group. $^{12}$

With regard to assessment of exposure and information bias, our investigators were dermatologists who knew whether subjects were controls or cases. The questionnaire, however, was standardised and did not contain open questions. Potential observer bias for assessment of toe-web interspaces was prevented by grouping the four categories in the questionnaire to two for the analysis.
Table 3 Comparison of number of toe-web intertrigo in cases and controls

\begin{tabular}{|c|c|c|c|c|c|}
\hline \multirow[b]{2}{*}{ Side } & \multirow{2}{*}{$\begin{array}{c}\text { No of affected } \\
\text { toe-web } \\
\text { interspaces } \\
\text { on one foot* }\end{array}$} & \multirow{2}{*}{$\begin{array}{c}\text { No }(\%) \text { of } \\
\text { cases } \\
(n=129)\end{array}$} & \multirow{2}{*}{$\begin{array}{c}\text { No }(\%) \text { of } \\
\text { controls } \\
(\mathrm{n}=294)\end{array}$} & \multicolumn{2}{|c|}{ Odds ratio $(95 \% \mathrm{CI})$} \\
\hline & & & & Adjusted $†$ & Adjusted‡ \\
\hline \multirow[t]{5}{*}{ Ipsilateral } & 0 & $43(34)$ & $216(77)$ & 1 & 1 \\
\hline & 1 & $16(13)$ & $19(7)$ & $4.9(2.2$ to 10.8$)$ & 6.5 (2.5 to 16.9$)$ \\
\hline & 2 & $25(20)$ & $25(9)$ & 6.1 (3.1 to 11.9$)$ & 8.9 (3.7 to 21.4) \\
\hline & 3 & $18(14)$ & $12(4)$ & 8.3 (3.6 to 19.2) & 12.5 (4.0 to 39.3 ) \\
\hline & 4 & $24(19)$ & $9(3)$ & $16.0(6.7$ to 38.3$)$ & 19.5 (5.4 to 70.2 ) \\
\hline \multirow[t]{5}{*}{ Contralateral } & 0 & $60(48)$ & $211(75)$ & 1 & 1 \\
\hline & 1 & $17(14)$ & $25(9)$ & 2.6 (1.3 to 5.3$)$ & $0.6(0.2$ to 1.6$)$ \\
\hline & 2 & $20(16)$ & $31(11)$ & 2.5 (1.3 to 4.7$)$ & $0.5(0.2$ to 1.3$)$ \\
\hline & 3 & $14(11)$ & $7(3)$ & 6.9 (2.6 to 18.4$)$ & $0.8(0.2$ to 2.9$)$ \\
\hline & 4 & $15(12)$ & $7(3)$ & 8.3 (3.2 to 21.7 ) & $0.8(0.2$ to 3.3$)$ \\
\hline
\end{tabular}

${ }^{*}$ Toe-web interspace considered affected if frankly abnormal or fissurated; doubtful cases considered normal. †Adjusted for age, sex, and hospital.

$\ddagger$ Adjusted for age, sex, hospital, and number of toe-web intertrigo on opposite foot.

Finally, major confounders were taken into account by matching factors and by adjustment during analysis. For lateralised factors, we controlled for confusion bias for presence or absence of a risk factor on the opposite leg by specific analyses.

\section{Risk factors for erysipelas of the leg}

Local factors seemed to be the most important risk factors for erysipelas of the leg. Lymphoedema showed the greater risk, which was present in $18 \%$ of our cases-more than in most, ${ }^{46}{ }^{8}$ but not all, ${ }^{13}$ prior series. Such a discrepancy may be due to the retrospective collection of data in prior studies or to differences in the definition of lymphoedema or lymphatic impairment. For most authors, lymphatic impairment plays a major role in the pathophysiology of erysipelas of the leg. ${ }^{13-15}$

A site of entry was found in almost all cases and could be considered as a requisite factor for the occurrence of a erysipelas of the leg. Toe-web intertrigo is mostly due to fungal infection (Tinea pedis), and its prevalence in the general population is probably higher than $10 \% .{ }^{16}$ In previous case series of erysipelas of the leg, prevalence of intertrigo ranged between $6 \%$ and $26 \%,{ }^{4-8}$ so whether toe-web intertrigo was a risk factor for erysipelas of the leg in these series was debatable. We showed a strong association between toe-web intertrigo and erysipelas of the leg. Toe-web intertrigo could have been linked to another general factor (for example, lack of hygiene) not listed in the questionnaire. The analysis with adjustment for intertrigo on the opposite foot ruled out such a hypothesis, and we concluded that intertrigo plays an exclusively local role. The credibility of the association between toe-web intertrigo and erysipelas of the leg is reinforced by the strength of the association and the relation between dose and effect (the odds ratios increased with the number of toe-web intertrigo on one foot). Because we systematically searched for toe-web intertrigo this may have explained its high prevalence $(66 \%)$ in our study; a similar prevalence among cases was found in a prospective study. ${ }^{17}$ In our study, traumatic wound and leg ulcer were also strong risk factors for erysipelas of the leg. Lack of association with pressure ulcer and excoriated leg dermatosis may have been due to a lack of power, given the small number of patients exposed to risk. 
Key messages

- Local factors are potential risks for erysipelas of the leg

- Lymphoedema and disruption of the cutaneous barrier exhibit the highest relative risk

- Toe-web intertrigo has a high population attributable risk, and its detection and treatment may prevent up to $60 \%$ of cases of erysipelas of the leg

Venous insufficiency was a significant risk factor for erysipelas of the leg although not as significant as the other risk factors. The definition of venous insufficiency is debatable, and as we chose ours on the basis of clinical findings only, this may have led to its underestimation. Interestingly, being overweight was the only general risk factor associated with erysipelas of the leg. In previously published case series, diabetes was present in $4.6 \%$ to $15 \%$ of cases $^{4-8}$ and therefore was suspected as a risk factor. Our study had enough power $(>80 \%)$ to detect an odds ratio $>2.5$ for prevalence of diabetes among controls. Similarly, we observed no association with alcohol misuse. Thus our findings exclude a strong association between either diabetes or alcohol misuse and erysipelas of the leg. These conclusions, however, do not apply to necrotic cellulitis, which we excluded from our study.

\section{Consequences in clinical practice}

The major concern in long term clinical management of patients with erysipelas of the leg is prediction and prevention of recurrences. As we restricted the analysis, because of methodological concerns, ${ }^{10}$ to cases with newly diagnosed erysipelas of the leg, we did not specifically study risk factors for recurrences. The prevalence of risk factors, however, was shown to be similar in cases of recurrence and first episodes, and it can be reasonably assumed that a patient with strong risk factors for a first episode also has a strong risk for recurrence if these factors remained unchanged. The prevention of recurrence is currently based on long term prophylactic antibiotherapy. ${ }^{18}{ }^{19}$ Toe-web intertrigo is highly prevalent in the population, and the high population attributable risk of toe-web intertrigo in our study suggests that suppression of this factor would result in a dramatic decrease in incidence of erysipelas of the leg. The importance of treating toe-web intertrigo has been previously acknowledged ${ }^{20}{ }^{21}$ but never assessed in a quantitative way. In contrast to leg ulcers or traumatic wounds, toe-web intertrigo is quite easy to avoid by detection and treatment. We therefore suggest that screening for, and treatment of, toe-web intertrigo should be a priority in subjects at high risk of erysipelas of the leg or in whom avoidance of recurrences is critical. Whether this strategy is sufficient alone or requires antibiotic prophylaxis needs to be investigated.

We thank the investigators who helped to collect the data: S Chauchaix (Tours), P M Dang (Limoges), H Dega (Paris), N Menard (Brest), and C Michel (Colmar). We thank L Mandereau for her help in the conditional analysis and P de La Salmonière for her critical review of the manuscript.
Contributors: All the authors, members of the RED (Réseau d'Epidémiologie en Dermatologie), participated in the protocol design, analysis, interpretation of the data, and writing of the paper. All of them, except AD and SB-G, recruited cases and controls. Furthermore, J-CR had the original idea for the present study and built the protocol design and questionnaire with SB-G. HB coordinated the seven participating centres, collected all the data, and set up the database. AD, under SB-G's supervision, conducted the statistical analysis, interpreted the data, and wrote the draft and the final version of the paper. SB-G will act as guarantor for the paper.

Funding: None.

Competing interests: None declared.

1 Bisno AL, Stevens DL. Streptococcal infections of skin and soft tissues. N Engl J Med 1996;334:240-5.

2 Bernard P. Dermo-hypodermal bacterial infections. Current concepts. Eur J Med 1992;1:97-104.

3 Bernard P, Bedane C, Mounier M, Denis F, Catanzano G, Bonnetblanc JM. Streptococcal cause of erysipelas and cellulitis in adults. A microbiologic study using a direct immunofluorescence technique. Arch Dermatol 1989:125:779-82.

4 Crickx B, Chevron F, Sigal-Nahum M, Bilet S, Faucher F, Picard C, et al. Erysipèle: Données épidémiologiques, cliniques et thérapeutiques. Ann Dermatol Venereol 1991;118:11-6.

5 Ronnen M, Suster S, Schewach-Millet M, Modan M. Erysipelas: changing faces. Int J Dermatol 1985;24:169-72.

6 Jorup-Rönström C. Epidemiological, bacteriological and complicating features of erysipelas. Scand J Infect Dis 1986;18:519-24.

7 Chartier C, Grosshans E. Erysipelas: an update. Int I Dermatol 1996;35:779-81.

8 Eriksson B, Jorup-Rönström C, Karkkonen K, Sjöblom AC, Holm SE. Erysipelas: clinical and bacteriologic spectrum and serological aspects. Clin Infect Dis 1996;23:1091-8.

9 Bush B, Shaw S, Cleary P, Delbanco T, Aronson M. Screening for alcohol abuse using the CAGE questionnaire. Am J Med 1987;82:231-5.

10 Breslow NE, Day NE, eds. Statistical methods in cancer research. Lyons: International Agency for Research on Cancer Scientific Publications, 1980.

11 Bruzzi P, Green SB, Byar DP, Brinton LA, Schairer C. Estimating the population attributable risk for multiple risk factors using case-control study. Am J Epidemiol 1985;122:904-14.

12 Wacholder S, Silverman DT, McLaughlin JK, Mandel JS. Selection of controls in case-control studies. II. Types of controls. Am J Epidemiol 1992;135:1029-41.

13 Duvanel T, Auckenthaler R, Rohner P, Harms M, Saurat JH. Quantitative cultures of biopsy specimens from cutaneous cellulitis. Arch Intern Med 1989;149:293-6.

14 Hook EW. Acute cellulitis. Arch Dermatol 1987;123:460-1.

15 Sachs MK. Cutaneous cellulitis. Arch Dermatol 1991;127:493-6.

16 Rogers D, Kilkenny M, Marks R. The descriptive epidemiology of tinea pedis in the community. Australas J Dermatol 1996;37:178-84.

17 Semel JD, Goldin H. Asociation of athlete's foot with cellulitis of the lower extremities: diagnostic value of bacterial cultures of ipsilateral space samples. Clin Infect Dis 1996;23:1162-4.

18 Sjöblom AC, Eriksson B, Jorup-Rönström C, Karkkonen K, Lindqvist M. Antibiotic prophylaxis in recurrent erysipelas. Infection 1993;21:390-3.

19 Kremer M, Zuckerman R, Avraham Z, Raz R. Long-term antimicrobial therapy in the prevention of recurrent soft-tissue infections. I Infect 1991;22:37-40.

20 Greenberg J, DeSanctis RW, Mills RM Jr. Vein-donor-leg cellulitis after coronary artery bypass surgery. Ann Intern Med 1982;97:565-6.

21 Baddour LM, Bisno AL. Recurrent cellulitis after coronary bypass surgery. Association with superficial fungal infection in saphenous venectomy limbs. JAMA 1984;251:1049-52.

(Accepted 5 March 1999)

\section{Endpiece \\ Women's complaints}

Diseases called women's: the womb is the cause of all diseases, for wherever the womb has moved from its proper place it causes illness, whether it goes forwards or whether it goes back. When the womb has moved but does not project its opening so as to touch the walls of the vagina, this is a very trivial complaint.

Hippocrates, Places of Man, edited and translated by Elizabeth M Craik, 1998

Submitted by Ann Dally, Wellcome Institute for the History of Medicine 